\title{
Superharmonic Resonances of Parametricly Excited Gear System Solved by Homotopy Analysis Method
}

\author{
Wen Jianmin, You Bindi*, Zhao Jijun, Sun Jianhua and Yu Guangbin \\ School of Naval Architecture and Ocean Engineering, Harbin Institute of Technology, \\ 264209 Weihai, China \\ *youbindi@hit.edu.cn
}

\begin{abstract}
An analytical technique, namely the homotopy analysis method (HAM), is applied to solve periodic solutions for superharmonic resonances of nonlinear oscillations with parametric excitation. Unlike perturbation methods, HAM does not depend on any small physical parameters at all. Thus, it is valid for both weakly and strongly nonlinear problems. Besides, different from all other analytic techniques, the HAM provides us a simple way to adjust and control the convergence region of the series solution by means of an auxiliary parameter $h$. In this paper, periodic analytic approximations for superharmonic resonances of nonlinear oscillations with parametric excitation are obtained by using the HAM, which agree well with numerical results. This article shows that the HAM is a powerful and effective technique for nonlinear dynamical systems.
\end{abstract}

Keywords: Nonlinear Oscillations, Parametrick Excitation, Homotopy Analysis Method (HAM), Superharmonic Resonances?

\section{Introduction}

Exact solutions of differential equations are rare in many branches of fluid mechanics, solid mechanics, and physics because of nonlinearity, inhomogeneity, variable coefficients, and so n. Hence, it is necessary to determine approximate solutions of complicated nonlinear differential equations by using some analytical techniques. Foremost among the analytic techniques are the methods of perturbations (asymptotic expansions in terms of a small/large parameter [1, 2]. Because many nonlinear problems do not contain such a small/large parameter named perturbation quantity, the artficial small parameter method, the $\delta$-expansion method and Adomian's decomposition method were developed [3-5]. In 1992, Liao employed the basic ideas of the homotopy in topology to propose a general analytic method for nonlinear problems, namely the homotopy analysis method (HAM)[6]. Different from all other analytic techniques, the HAM provides us with a simple way to adjust and control the convergence region of approximate series solutions [7]. HAM has been successfully applied to solve many types of nonlinear problems [8-15].In this paper, the basic idea of HAM is used to solve nonlinear oscillations which often appear in some parametricly excited gear systems[16], governed by

$$
\frac{d^{2} x}{d t^{2}}+2 \mu \frac{d x}{d t}+k_{e}(t) f(x)=F_{m}+F_{a t} \cos \left(\omega_{a T} t+\varphi_{T}\right)+F_{a h} \omega_{e h}^{2} \cos \omega_{e h} t
$$

* Corresponding Author 
where $k_{e}(t)$ is time-varying mesh stiffness, $f(x)$ is a nonlinear displacement function which includes gear backlash and can be defined by equation (2). $\mu$ is the damping coefficient, $F_{m}$ is the mean load of the gear pair. $F_{a T}$ and $F_{a h}$ are the amplitudes of external and internal excitations. Parameters $\omega_{e}, \omega_{e T}$ and $\omega_{e h}$ are the frequencies of the parametric, external and internal excitations, respectively.

$$
k_{e}(t)=1+\sum_{j=1}^{5} B_{j} \cos \left(j \omega_{e} t+\varphi_{j}\right), \quad f(x)=a_{1} x+a_{3} x^{3}+a_{5} x^{5}+a_{7} x^{7}
$$

For the sake of simplicity, the equation (1) can be rewritten as

$$
\frac{d^{2} x}{d t^{2}}+[1+\varepsilon \cos (\gamma t)]\left(\alpha x+\beta x^{3}\right)=0
$$

where $x(t)$ is an unknown real function, $\varepsilon, \alpha, \beta, \gamma$ are known physical parameters. Here, we are interested in the periodic oscillations with the frequency

$$
\omega=n \gamma \quad(n=1,2,3, \cdots)
$$

In other words, given $\varepsilon, \alpha, \beta, \gamma$ and the frequency described above, the HAM is applied to find the corresponding unknown amplitude $A$, such that

$$
x(0)=A 6 \quad x(0)=8
$$

\section{Basic Idea of HAM}

Assuming that the solution of Eq. (3) and (5) is periodic with the frequency $\omega$ defined by (4). Then, writing

Eq. (3) becomes

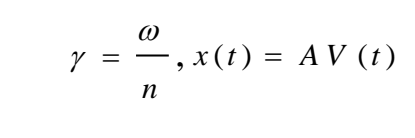

$$
\text { (t) }+\left(1+\varepsilon \cos \frac{\omega t}{n}\right)\left[\alpha V(t)+\beta A^{2} V^{3}(t)\right]=0
$$

subject to the initial conditions

$$
V(0)=1, V^{\prime}(0)=0
$$

Accor ing to the initial conditions (8) and the nonlinear term in Eq. (7), the periodic solution of $V(t)$ with the given frequency $\omega$ can be expressed by

$$
V(t)=\sum_{k=1}^{+\infty} c_{k} \cos k \omega t
$$

where $c_{k}$ is a coefficient to be determined. This provides us with the so-called solution expression of $V(t)$, which plays an important role in the frame of the HAM, as shown later. For the sake of simplicity, using the transformation $\tau=\omega t$ and $V(t)=u(\tau)$, Eqs. (7) and (8) are rewritten as 


$$
\begin{gathered}
\omega^{2} u^{\prime \prime}(\tau)+\left(1+\varepsilon \cos \frac{\tau}{n}\right)\left[\alpha u(\tau)+\beta A^{2} u^{3}(\tau)\right]=0 \\
u(0)=1, u^{\prime}(0)=0
\end{gathered}
$$

According to the solution expression (9), $u(\tau)$ can be expressed by

$$
u(\tau)=\sum_{k=1}^{+\infty} c_{k} \cos k \tau
$$

According to (11) and (12), it is obvious for us to choose such an initial guess

$$
u_{0}(\tau)=\cos \tau
$$

According to (12), obviously, we should choose the auxiliary linear operator

$$
L[\Phi(\tau, q)]=\omega^{2}\left[\frac{\partial^{2} \Phi(\tau, q)}{\partial \tau^{2}}+\Phi(\tau q)\right]
$$

which has the property

$$
L\left(C_{1} \sin \tau+C_{2} \cos \tau\right)=0
$$

for any integration constants $C_{1}$ and $C_{2}$. We define a nowlinear operator

$$
N[\Phi(\tau, q), \Lambda(q)]=\omega^{2} \frac{\partial^{2}[\Phi(\tau, q)]}{\partial \tau^{2}}\left(1-\varepsilon \cos \frac{\pi}{n}\left(\alpha \Phi(\tau, q)+\beta \Lambda^{2}(q) \Phi^{3}(\tau, q)\right]\right.
$$

where $q \in[0,1]$ is an embedding parameter. $\Phi(\tau, q)$ is a kind of mapping of the unknown function $u(\tau)$, and $\Lambda(q)$ is a kind of mapping of the unknown amplitude $A$. Then, we construct the so-called zero-order deformation equation

subject to the initial conditions

$$
(1-q) L\left[\Phi(\tau, q)-u_{0}(\tau)\right]=h q N[\Phi(\tau, q), \Lambda(q)]
$$

$$
\Phi(0, q)=1,\left.\quad \frac{\partial \Phi(\tau, q)}{\partial \tau}\right|_{\tau=0}=0
$$

When $q=0$, the solution of Eqs. (14) and (15) is

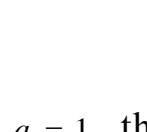

$$
\Phi(\tau, 0)=u_{0}(\tau)
$$

When $q=1$, the zero-order deformation equations (17) and (18) are equivalent to the original equations (10) and (11), provided

$$
\Phi(\tau, 1)=u(\tau), \Lambda(1)=A
$$

Therefore, as the embedding parameter $q$ increases from 0 to $1, \Phi(\tau, q)$ varies from the initial guess $u_{0}(\tau)=\cos \tau$ to the unknown solution $u(\tau)$ of Eqs (10) and (11). Likewise, $\Lambda(q)$ varies from the initial guess $A_{0}$ to the unknown amplitude $A$. Note that the zero-order deformation equation (17) contains a nonzero auxiliary parameter $h$. 
Assuming that $h$ is chosen so properly that the zero-order deformation equations (17) and (18) have solutions in the whole region $q \in[0,1]$, and besides, there exist

$$
u_{m}(\tau)=\left.\frac{1}{m !} \frac{\partial^{m} \Phi(\tau, q)}{\partial q^{m}}\right|_{q=0}, \quad A_{m}=\left.\frac{1}{m !} \frac{d^{m} \Lambda(q)}{d q^{m}}\right|_{q=0}
$$

For any $m \geq 1$. Then, by means of Taylor series and using (16), we have

$$
\Phi(\tau, q)=u_{0}(\tau)+\sum_{m=1}^{+\infty} u_{m}(\tau) q^{m}, \quad \Lambda(q)=A_{0}+\sum_{m=1}^{+\infty} A_{m} q^{m}
$$

Assuming that $h$ is so properly chosen that the power series (22) converge at $q=\hat{p}$, using (22), we have the series solutions

$$
u(\tau)=u_{0}(\tau)+\sum_{m=1}^{+\infty} u_{m}(\tau) \quad A=A_{0}+\sum_{m=1}^{+\infty} A_{\text {感 }}
$$

For the sake of simplicity, define the vectors

$$
\vec{u}_{m}=\left\{u_{0}(\tau), u_{1}(\tau), u_{2}(\tau), \cdots, u_{m}(\tau)\right\}, \vec{A}_{m}=\left\{A_{0}, A_{V} A_{2}, \cdots, A_{m}\right\} .
$$

Differentiating the zero-order deformation equations (17) and (18) $\mathrm{m}$ times with respect to the embedding parameter $q$, then dividing them by $m !$, and finally setting $q=0$, we have the so-called $m$ th-order deformation equation

subject to the initial conditions

$$
L\left[u_{m}(\tau)-\chi_{m} u_{m-1}(\tau)\right]=\vec{c}^{R} \vec{r}_{m}\left(\vec{u}_{m-1}, \vec{A}_{m-1}\right)
$$

$$
+\left(1+\varepsilon \cos \frac{\tau}{h}\left(\tau \alpha u_{m-1}+\beta \sum_{k=0}^{m-1}\left(\sum_{i=0}^{k} A_{i} A_{k-i}\right)\left[\sum_{j=0}^{m-1-k} u_{j}(\tau) \sum_{r=0}^{m-1-k-j} u_{r}(\tau) u_{m-1-k-j-r}(\tau)\right]\right.\right.
$$

and $\chi$

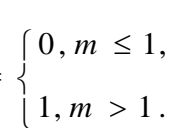

Note that both $u_{m}(\tau)$ and $A_{m-1}$ are unknown, but we have now only one equation (24) for $u_{m}(\tau)$. Thus, another algebraic equation must be given so as to determine $A_{m-1}$ . It is found that the right-hand side of the $m$ th-order deformation equation (24) is expressed by

$$
h \vec{R}_{m}\left(\vec{u}_{m-1}, \vec{A}_{m-1}\right)=b_{m, 0}\left(\vec{A}_{m-1}\right)+\sum_{k=1}^{\mu_{m}} b_{m, k}\left(\vec{A}_{m-1}\right) \cos (k \tau)
$$


where $b_{m, k}\left(\vec{A}_{m-1}\right)$ is a coefficient, $\mu_{m}$ is a positive integer dependent on the order $m$. According to the property (15) of the auxiliary linear operator $L$, when $b_{m, 1}\left(\vec{A}_{m-1}\right) \neq 0$, the solution of the $m$ th-order deformation equation (24) and (25) contains the so-called secular term $\tau \cos \tau$, which however disobeys the solution expression (9). To avoid the secular terms, we must enforce

$$
b_{m, l}\left(\vec{A}_{m-1}\right)=0
$$

which provides us another algebraic equation for $A_{m-1}$. Then, the general solution of equation (24) reads

$$
u_{m}(\tau)=\chi_{m} u_{m-1}(\tau)+\frac{h}{\omega^{2}} \sum_{k=2}^{\mu_{m}} \frac{b_{m, k}\left(A_{m-1}\right)}{\left(1-k^{2}\right)} \cos (k \tau)+C_{1} \cos \tau+C_{2} \sin \tau
$$

where the integration constants $C_{1}, C_{2}$ are determined by the initial conditions (25). The $N$ th-order approximation is given by

$$
u(\tau) \approx u_{0}(\tau)+\sum_{m=1}^{N} u_{m}(\tau), \quad A \approx A_{0}+\sum_{m}^{N} A_{m}
$$

Note that $\gamma$ is given, and thus $\omega=n \gamma$ determined by the given positive integer $n$ is known. For example, $\omega=\gamma$ when $n=1,0=2 \gamma$ when $n=2, \omega=3 \gamma$ when $n=3$, and so on.

\section{Result Analysis}

For given $\varepsilon, \alpha, \beta, \gamma$ and $n$, the periodic solution with the known frequency $\omega=n \gamma$ and the corresponding unknown amplitude $A$ can be determined by the analytic approach mentioned above. Note that there exjst auxiliary non-zero parameter $h$, which provides us with a simple way to ensure the convergence of solution series, as mentioned by Liao [6-12] and other authors [13-15. For example, let us consider the case of $\alpha=1, \beta=4, \gamma=10$, $\varepsilon=0.01$, and $n=1(i \mathrm{e},=10)$. Such kind of excitation is often called the primary parametric resonance Obviously, the amplitude $A$ contains the auxiliary non-zero parameter $h$. As suggested by Liao, one can plot the $A-h$ curve to determine the so-called valid region of $h$, as shown in Figure 1. Obviously, the series of $A$ converges when $0<h<0.06$. For instance, when $h=0.015$, we have the convergent result $A \approx 5.85329$, as shown in Table 1 . The corresponding 2th-order approximation of $x(t)$ agrees well with the numerical result, as shown in Figure 2.

Similarly, in case of $\alpha=1, \beta=4, \gamma=10, \varepsilon=0.01$, and $n=2$ (i.e., $\omega=20$ ), we obtain the convergent amplitude $A \approx 11.7536$ by means of $h=0.015$. And it is a little surprise that even the corresponding 2nd HAM approximation agrees well with the numerical ones, as shown in Figure 3. 
Table 1. The Analytic Approximations of $A$ by Means of $h=0.015$

\begin{tabular}{lcl}
\hline Order N & $A$ & Error: $(A \mathrm{n}-A \mathrm{n}-1) / A \mathrm{n}$ \\
\hline 1 & 5.76 & 0.00266 \\
2 & 5.77 & 0.00243 \\
3 & 5.79 & 0.00221 \\
4 & 5.80 & 0.00200 \\
5 & 5.81 & 0.00180 \\
6 & 5.82 & 0.00162 \\
7 & 5.83 & 0.00146 \\
8 & 5.83 & 0.00131 \\
9 & 5.84 & 0.00119 \\
10 & 5.85 & 0.00107 \\
11 & 5.85 & 0.00097 \\
\hline
\end{tabular}

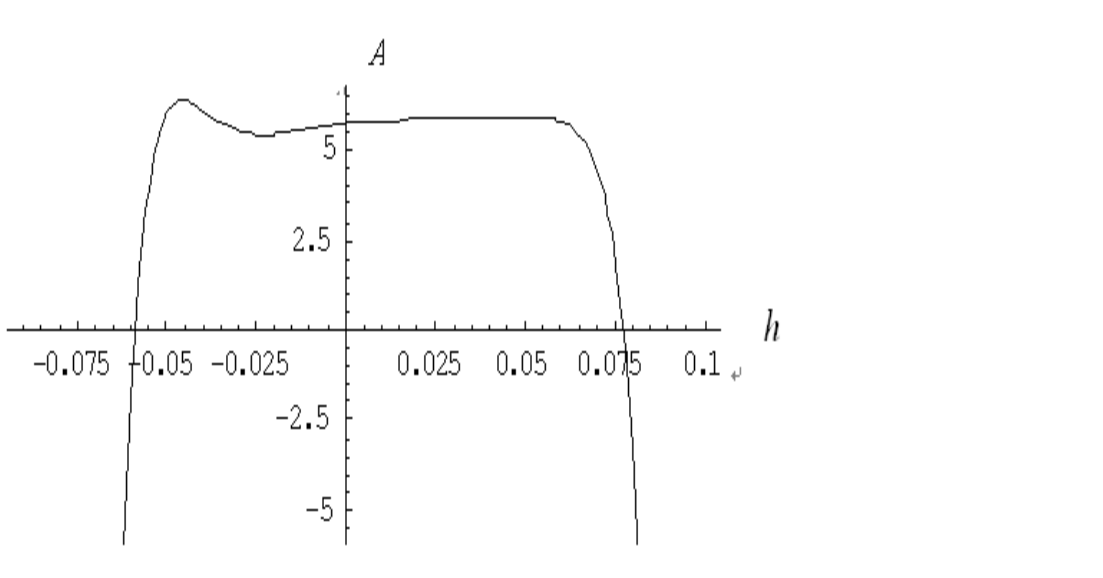

Figure 1. The 11th-order Approximation of $A$ versus $h$ in Case of $\alpha=1, \beta=4$, $\gamma=(10), \varepsilon=0.0$, , and $n=1$ (i.e., $\omega=10$ )

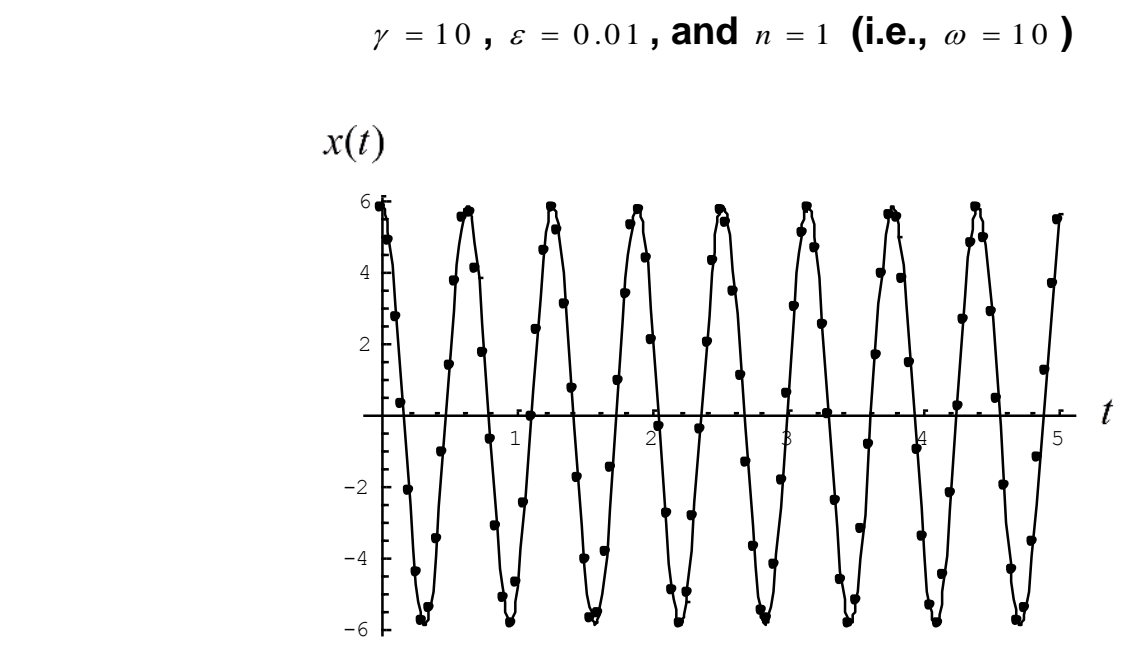

Figure 2. Comparison of the 2nd-order HAM Approximation of $x(t)$ with the Numerical Solution in Case of $h=0.015, \alpha=1, \beta=4, \gamma=10, \varepsilon=0.01$, and $n=1$ (i.e. $\omega=10$ ). Solid Line: HAM result; circle: Numerical Solution 


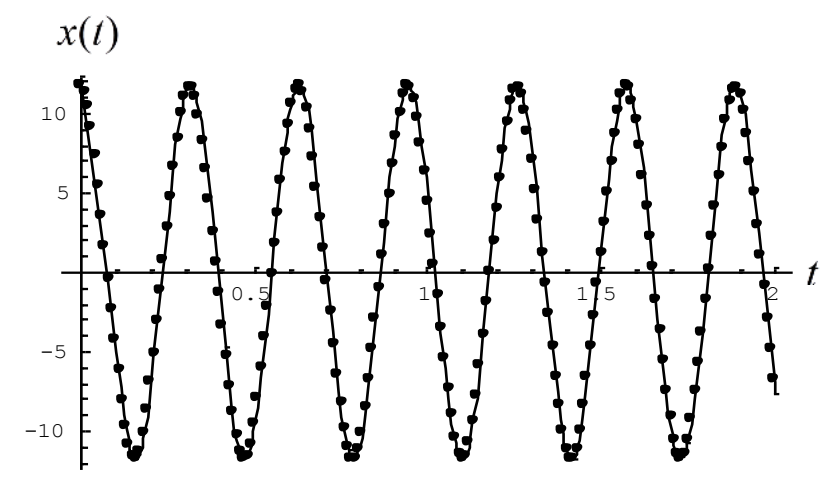

Figure 3. Comparison of the 2nd-order HAM Approximation of $x(t)$ with the Numerical Solution in Case of $h=0.015, \alpha=1, \beta=4, \gamma=10, \varepsilon=0.01$, and $n=2$ (i.e. $\omega=20$ ). Solid Line: HAM Result; Circle: Numerical Solution

In a similar way, note that when $n=2,3,4$, etc., the natural frequencies of oscillation are 2 , 3 and 4 times of the frequency $\gamma$ of parametric excitation, respectively. Such cases can be called superharmonic resonances which are very important in engineering.

\section{Conclusions}

In this paper, an analytical technique namely the homotopy analysis method (HAM), is applied to obtain the periodic solutions for superharmonic resonances of nonlinear oscillations with parametric excitation, For given physical parameters and the frequency of superharmonic resonances, the convergent series solutions of the corresponding amplitude $A$ and $x(t)$ are explicitly obtained, which agree well with the numerical results. All of these verity that the HAM is valid for superharmonic resonances of nonlinear oscillation systems with parametric excitation.

Indeed, the homotopy analysis method (HAM) has many advantages. Different from all other analytic techniques, it provides us with a simple way to adjust and control the convergence egion of approximate series solutions[9-22]. The current work illustrates that the HAM is indeed a powerfund versatile analytical technique for most types of nonlinear problems, and might have many applications in science and engineering.

\section{Acknowledgements}

This paper is based on the Project(Approval No. 51275108 and 51205079) supported by National Natural Science Foundation of China, the Project (Approval No. ZR2012EEM018) supported by Shandong Provincial Natural Science Foundation and the Project (HIT. NSRIF. 2011107 and HIT. NSRIF. 2015110) supported by Natural Scientific Research Innovation Foundation in Harbin Institute of Technology, and the Key Program of National Natural Science Foundation of Heilongjiang (No.ZD201309), and the Major International Joint Research Program of China (Grant No. 2014DFB70120). Furthermore, we would like to express my sincere thanks to Professor Liao for his valuable comments and discussions.

\section{References}

[1] A. H. Nayfeh. Introduction to Perturbation Techniques. John Wiley \& Sons, New York (1981).

[2] A. H. Nayfeh. Problems in Perturbation. John Wiley \& Sons (1985). 
[3] A. M. Lyapunov. General Problems on Stability of Motion. Taylor \& Francis, London (1992).

[4] A. V. Karmishin, A. I. Zhukov, and V. G. Kolosov. Methods of Dynamics Calculation and Testing for ThinWalled Structures. Msshinostroyenie, Moscow (1990).

[5] D. Kaya. Solitary wave solutions for a generalized Hirota-Satsuma coupled KdV equation. Applied Mathematics and Computation, vol. 147, (2004), pp. 69-78.

[6] S. J. Liao, "The Proposed Homotopy Analysis Technique for the Solution of Nonlinear Problems", PhD Thesis, Shanghai Jiaotong University (1992).

[7] S. J. Liao, "Beyond Perturbation: Introduction to the Homotopy Analysis Method", Chapman \& Hall/CRC, Boca Raton (2003).

[8] S. J. Liao, "A uniformly valid analytic solution of 2D viscous flow past a semi-infinite flat plate", Journal of Fluid Mechanics, vol. 385, (1999), pp. 101-128.

[9] S. J. Liao and A. Campo, "Analytic solutions of the temperature distribution in Blasius viscous flow problems. Journal of Fluid Mechanics, vol. 453, (2002), pp. 411-425.

[10] S. J. Liao, "Series solutions of unsteady boundary-layer flows over a stretching flat plate", Studies in Applied Mathematics, vol. 117, (2006), pp. 239-263.

[11] S. J. Liao and E. Magyari. Exponentially decaying boundary layers as limiting cases of families of algebraically decaying ones. Z. angew. Math. Phys. (ZAMP), vol. 57, (2006), pp. 777-792.

[12] S. J. Liao, "A new branch of solutions of boundary-layer flows over an impermeable stretched plate", Int. J. Heat and Mass Transfer, vol. 48, (2005), pp. 2529-2539.

[13] T. Hayat and M. Sajid, "On Analytic Solution for Thin Film Flow of a Fourth Grade Fluid down a Vertical Cylinder”, Physics Letters A, vol. 361, (2007), pp. 316-322.

[14] S. Abbasbandy, "The Application of Homotopy Analysis Method to Solve a Genefalized Hirota-Satsuma Coupled KdV Equation", Physics Letters A, vol. 361, (2007), pp. 478-483.

[15] F. M. Allan and M. I. Syam, "On the analytic solution of non-homogeneous Blasius problem", J. Computational and Applied Mathematics, vol. 182, (2005), pp. 362-371.

[16] Y. X. Wang and J. M. Wen, "Gear Method for Solying Differential Equations of Gear Systems", International Symposium on Instrumentation Science and Technology, Journal of Physics: Conference Series, vol. 48, (2006), pp. 143-148.

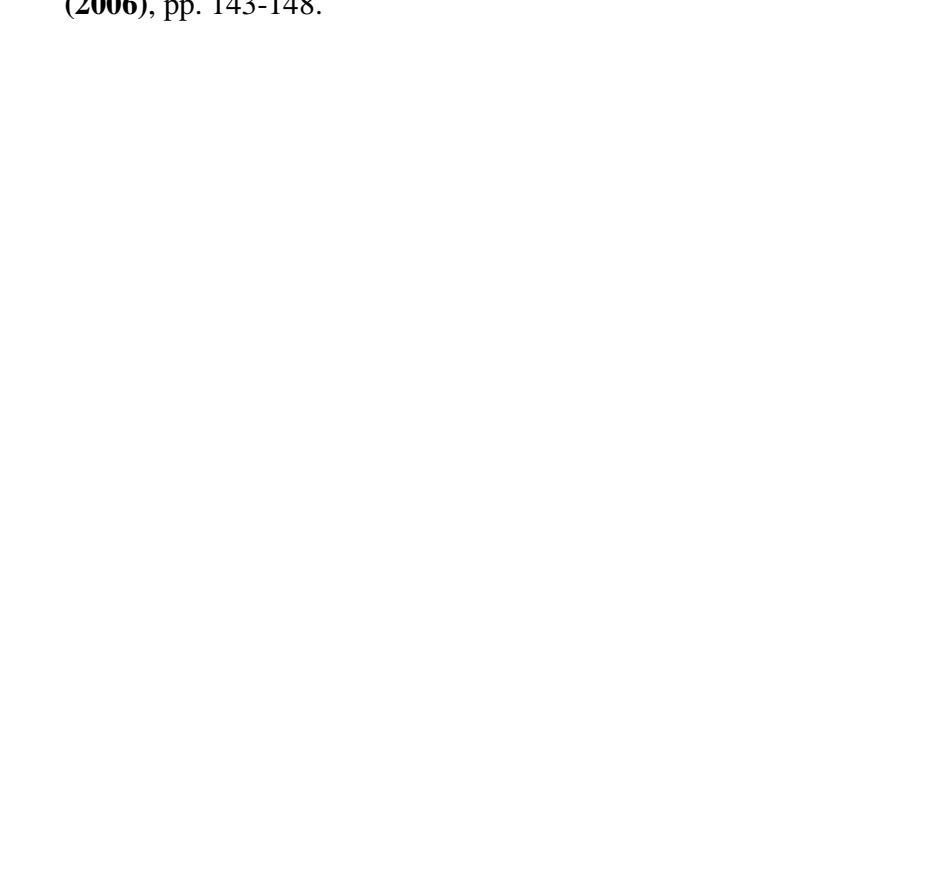

